\title{
Residual stresses in amorphous alumina films synthesized by ion beam assisted deposition
}

\author{
L. Parfitt \\ Department of Materials Science and Engineering, University of Michigan, Ann Arbor, \\ Michigan 48109-2104 \\ M. Goldiner \\ Department of Nuclear Engineering, University of Michigan, Ann Arbor, Michigan 48109-2104 \\ J.W. Jones and G. S. Was ${ }^{\text {a) }}$ \\ Department of Materials Science and Engineering, University of Michigan, Ann Arbor, \\ Michigan 48109-2104
}

(Received 25 August 1994; accepted for publication 12 December 1994)

A set of experiments was conducted to determine the origin of residual stresses in amorphous $\mathrm{Al}_{2} \mathrm{O}_{3}$ films formed by ion beam assisted deposition. Samples were deposited during bombardment by Ne, $\mathrm{Ar}$, or $\mathrm{Kr}$ over a narrow range of energies, $E$, and a wide range of ion-to-atom arrival rate ratios, $R$. Films were characterized in terms of composition, thickness, density, crystallinity, microstructure, and residual stress. Film composition was independent of ion beam parameters and residual stress was independent of thickness over the range $200-1200 \mathrm{~nm}$. Stress varied strongly with ion beam parameters and gas content. Residual stress and gas content saturated at a normalized energy of $\sim 20$ $\mathrm{eV} /$ atom or an $R$ of $\sim 0.05$. Where residual stress varied linearly with $R E^{1 / 2}$, results are consistent with an atom peening model, but saturation at high $R$ or $R E^{1 / 2}$ is inconsistent with such a model. Stress due to gas pressure in existing voids explains neither the functional dependence on gas content nor the magnitude of the observed stress. A probable explanation for the behavior of stress is gas incorporation into the matrix, where the amount of incorporated gas is controlled by trapping. (1) 1995 American Institute of Physics.

\section{INTRODUCTION}

The use of an ion beam during physical vapor deposition (PVD) is a powerful tool for controlling the structure and properties of deposited films. Ion beam assisted deposition (IBAD) is being intensively studied for applications in films and coatings with unique and controllable properties. Advantages include a high degree of control over film structure, microstructure, density, texture, and residual stress, which influence optical, electrical, transport, electrochemical, and mechanical properties of the film or coating. Recently, commercial products have appeared which utilize ion beam assisted deposition to achieve a degree of control over properties that is otherwise impossible by conventional deposition techniques. ${ }^{1,2}$ However, an understanding of the mechanisms by which structure and properties are altered by the ion beam is incomplete. For example, residual stresses formed during deposition are very important in determining coating integrity since high residual tensile or compressive stresses can initiate coating degradation in service. While IBAD has been shown to produce significant changes in both the magnitude and sign of residual stresses in deposited films, the mechanism(s) of residual stress generation by IBAD are not well established. This article presents the results of a study which shows that the incorporation of inert gas atoms into an amorphous alumina film during deposition is likely responsible for the observed changes in residual stress.

4)Also with Department of Nuclear Engineering; E-mail: gsw@umich.edu

\section{BACKGROUND}

Several models have been proposed to explain the origin of residual stress in deposited films and excellent reviews on the subject ${ }^{3.6}$ are available. These models fall broadly into two categories, those which relate to the development of a tensile stress and those which address the formation of a residual compressive stress. In the former category are models based on densification, grain growth, and phase changes. Residual tensile stresses are believed to result from densification or grain growth when structural relaxation lags the deposition rate. Structural relaxation occurs in order to minimize the free energy of the system and results in a reduction of the specific volume of the film as long as the film is unconstrained. However, if the film is geometrically constrained by the substrate, volumetric relaxation is inhibitcd and a residual stress (and strain) is produced in the film and substrate. Phase changes resulting in a different specific volume also produce tensile or compressive stresses depending upon the sign of the volume change.

Compressive residual stresses are less common in PVD films but are frequently observed in films formed by ion beam assisted deposition. Explanations proposed for their origin include thermal spikes, recoil implantation, and trapped bombardment gas. The issuc of residual stress modification by ion bombardment was first treated by Hirsch and Varga ${ }^{7}$ in an attempt to explain the improved adhesion of amorphous Ge films after ion bombardment. They postulated that "annealing" within the thermal spike of the incoming ion was responsible for the observations and that this effect becomes apparent at a value of ion current density which 
ensures that all of the Ge atoms are part of the rearrangement process in the thermal spike. Their primary evidence was the correlation between the empirical dependence of critical current density on ion energy $\left(I_{c} \propto E^{-3 / 2}\right)$, which was similar to that predicted by the Seitz and Koehler ${ }^{8}$ model $\left(I_{c} \propto E^{-5 / 3}\right)$. However, the authors made no attempt to provide a physical process to account for the observed reduction in intrinsic residual tensile stress.

Another mechanism is atom peening, proposed by Hoffman and Thornton ${ }^{9}$ and recently furthered by Windischmann. ${ }^{3,10}$ In this model, the forward momentum of the bombarding ion is transferred to the lattice which results in densification and the generation of compressive stresses below the surface. Windischmann uses Sigmund's knock-on linear cascade theory ${ }^{11}$ to determine the fractional number of atoms displaced from equilibrium, which he then relates to the volumetric distortional strain and stress by Hooke's law for a biaxial stress state. The resulting equation for stress is given as

$$
\sigma=A R E^{1 / 2} \delta Q
$$

where $\sigma$ is the film stress, $A$ is a constant, $E$ is the projectile energy, $R$ is the ion-to-atom arrival rate ratio, $Q$ is the elastic energy per mole and $\delta$ is defined as

$\delta=Z_{p}^{1 / 2} Z_{t}^{1 / 2} / U_{o}\left(1+M_{p} / M_{t}\right)^{1 / 2}\left(Z_{p}^{2 / 3}+Z_{t}^{2 / 3}\right)^{3 / 4}$,

where the $Z_{p, t}$ and $M_{p, t}$ are the atomic number and atomic mass of projectile and target, respectively, and $U_{n}$ is the sublimation energy of the target. Note that $\mathrm{Eq}$. (1) predicts a stress dependence on the projectile energy to the $1 / 2$ power and a linear dependence on the $R$ ratio, while the dependence upon the projectile mass is incorporated into $\delta$. Windischmann $n^{3,10}$ has shown that in many sputtered metal films, the linear relation between $\sigma$ and $\delta Q$ does indeed hold. However, quantitative verification of the magnitude of the proportionality coefficient has not been established. Additionally, verification of the dependence of stress on $R$ and $E$, separately has been hampered by their interdependence in a sputter deposition mode, from which the bulk of the data has been obtained.

A third mechanism by which ion bombardment can affect residual stress is through the incorporation of the bombardment gas into the film either in the matrix or in the form of gas-filled voids. This idea has been well studied, but conflicting experimental results have prevented a consensus on this mechanism. While Thornton has shown that the elastic strain in sputter deposited films does not change appreciably over a two-decade variation in argon content, ${ }^{12}$ recent data by Dietz et al. on $\mathrm{Al}^{13}$ and molecular-dynamics simulations by Fang et al. ${ }^{14}$ show that the incorporated gas either dominates or plays a significant role in the resulting stress state. Even Hoffman and Gaerttner ${ }^{15}$ found that gas entrapment was needed to explain the compressive stress transition in chromium films deposited onto glass. This paper seeks to clarify the origin of the residual stress in amorphous alumina synthesized by ion beam assisted deposition.

\section{EXPERIMENTAL PROCEDURE}

IBAD films of alumina were deposited in a vacuum chamber with a base pressure of $\sim 2 \times 10^{-8}$ Torr. The chamber contains two $6 \mathrm{~kW}$ electron beam guns and a $3 \mathrm{~cm}$ Kaufman ion gun. Depositions are conducted with the sample substrate normal to the evaporant and at an angle of $45^{\circ}$ to the ion beam. Films were made by electron beam evaporation of $99.99 \%$ pure $\mathrm{Al}_{2} \mathrm{O}_{3}$ at nominal deposition rates between 0.32 and $1.52 \mathrm{~nm} / \mathrm{s}$ to create film thicknesses ranging from 223 to $1209 \mathrm{~nm}$ as measured by a quartz crystal thickness monitor. Depositions were made with simultaneous ion bombardment by $\mathrm{Ne}, \mathrm{Ar}$, or $\mathrm{Kr}$ at energies between 325 and $700 \mathrm{eV}$. Ion current densities up to $265 \mu \mathrm{A} / \mathrm{cm}^{2}$ were measured using a Faraday cup attached to the copper specimen block. The minimum current density was $\sim 10 \mu \mathrm{A} / \mathrm{cm}^{2}$, below which the beam tended to become unstable. Ion-to-atom arrival rate ratios $(R)$ ranged from 0.003 to 0.19 and yielded normalized energies; $E_{n}=R E$, ranging from $\sim 1$ to $100 \mathrm{eV} /$ atom. The normalized energy is the average energy per deposited atom. Chamber pressure during deposition was in the range $2-5 \times 10^{-5}$ Torr, due primarily to the inert gas.

A thermocouple attached to the copper specimen block indicated that substrate heating of approximately $200^{\circ} \mathrm{C} \mathrm{oc}-$ curred for the highest ion currents and deposition rates, placing the deposition in Zone 1 of Thornton's sputter deposition version of the structure zone model. ${ }^{16}$ Three types of substrates were used for analysis purposes: a soda lime glass cover slip (150 $\mu \mathrm{m}$ thick) for residual stress, a graphite foil for determining composition by Rutherford backscattering spectroscopy (RBS), and two Si substrates for microstructure and thickness determination by transmission electron microscopy (TEM), x-ray diffraction (XRD), profilometry, and ellipsometry. Substrates were cleaned prior to deposition by ion bombardment at a beam voltage of $500 \mathrm{eV}$ and a beam current of $25 \mathrm{~mA}$.

Composition and stoichiometry were obtained from RBS using $\mathrm{He}^{++}$ions of energy $2.0 \mathrm{MeV}$. RBS profiles were fit using the computer program RUMP to yield the number of AI and $\mathrm{O}$ atoms $/ \mathrm{cm}^{2}{ }^{17}$ The film thicknesses were determined by two methods, profilometry, and ellipsometry. Profilometry was conducted on a Dektak IIA profilometer which traced a step created by partially masking a Si substrate during deposition. The measured step height was corrected for the loss of Si due to sputter cleaning using an empirically determined relation between the sputter depth and ion current and energy for this system. RBS and profilometry values were obtained from the edges of the $\mathrm{Si}$ and the graphite in closest proximity on the $\mathrm{Cu}$ block to minimize error due to thickness changes across the width of the $\mathrm{Cu}$ block. Thickness was also determined by ellipsometry using a He-Ne laser at a wavelength of $633 \mathrm{~nm}$. The density of the films was determined by three methods. In the first two, the areal atom density determined by RBS was divided by the film thicknesses as measured either by profilometry or ellipsometry. The third method utilized the index of refraction as measured by ellipsometry, in the following relation: ${ }^{18}$

$$
\rho=\left(n_{f}^{2}-1\right) /\left(n_{b}^{2}-1\right)
$$


where $\rho$ is the film density, $n_{f}$ is the index of refraction of the film, and $n_{b}$ is the index of refraction of bulk $\mathrm{Al}_{2} \mathrm{O}_{3}$, $n_{b}=1.774$.

Residual stress was determined by optical interferometry of films deposited onto glass cover slips. The shape of the cover slip was determined before and after deposition with reference to an optical flat by observation in monochromatic light. The resulting pattern of Newton's rings was photographed and the fringe spacing measured in orthogonal directions. Curvatures were determined by least squares fitting of parabolas to the recorded fringe spacings. The resulting quadratic coefficients, $k_{x}$ and $k_{y}$ are linearly related to the average stress by the formula: ${ }^{19}$

$$
\sigma=\frac{E h^{2}\left(\Delta k_{x}+\Delta k_{y}\right)}{6(1-\nu) t}
$$

where $E$ and $\nu$ are Young's modulus and Poisson's ratio of the cover slip, respectively, $h$ is the cover slip thickness, $t$ $(\ll h)$ is the thickness of the deposited layer (from profilometry), and the $\Delta k$ 's indicate the changes in the quadratic coefficients induced by the deposition. The values used for the modulus of elasticity, $E$, and Poisson's ratio, $v$ of soda lime glass were $70 \mathrm{GPa}$ and 0.22 , respectively. ${ }^{9}$

Thermal stress was estimated using the relation:

$$
\sigma_{t h}=E_{f}\left(\alpha_{f}-\alpha_{s}\right)\left(T_{d}-T_{m}\right),
$$

where $E_{f}$ is the elastic modulus of the film, $\alpha_{f}$ and $\alpha_{s}$ are the thermal expansion coefficients of the film and substrate, $T_{d}$ is the deposition temperature, and $T_{m}$ is the measurement temperature. The values used were $E_{f}=150 \mathrm{GPa},{ }^{20} \alpha_{f}=7 \times 10^{-6}$ $\mathrm{K}^{-1},{ }^{21} \alpha_{s}=9.2 \times 10^{-6} \mathrm{~K}^{-1},{ }^{22}$ and $\left(T_{d}-T_{m}\right)=200 \mathrm{~K}$ yielding a value of thermal (extrinsic) stress of $-0.066 \mathrm{GPa}$.

Structure and crystallinity were analyzed by transmission electron microscopy (TEM) using a JEOL 2000FX operated at $200 \mathrm{kV}$. Plan view sảmples were prepared by grinding the Si substrate and then either etching with a solution of acetic, nitric, and hydrofluoric acid, or ion milling. Of the samples analyzed, one was deposited at $R=0$ and the other three samples were deposited at a nominal $R$ ratio of 0.1 using a $\mathrm{Ne}$, Ar, or $\mathrm{Kr}$ ion beam. Cavity size and number density were determined using a densitometer trace from a micrograph at a magnification of $200000 \times$, which gave a focal depth approximately equal to the void diameter.

The cavity size distribution was determined by small angle $\mathrm{x}$-ray scattering (SAXS) using a Kratky camera arrangement in which the $\mathrm{x}$-ray source, sample, and detector are colinear and the sample is situated $8.6 \mathrm{~cm}$ from the source and $17.5 \mathrm{~cm}$ from the detector. A slit, $500 \mu \mathrm{m} \times 2.4$ $\mathrm{cm}$, is placed just in front of a position-sensitive wire detector. The $\mathrm{x}$-ray source is $\mathrm{Cu} K \alpha$ radiation, and it is operated at $40 \mathrm{kV}$ and $60 \mathrm{~mA}$. Void size and density were determined from Guinier's law: ${ }^{23}$

$$
I(k)=I(0) \exp \left(-k^{2} R_{g}^{2 / 3}\right),
$$

and

$$
I(0)=I_{p} r_{e}^{2} \Delta n^{2} / L^{2},
$$

where $I(k)$ is the scattering intensity as a function of the diffraction vector, $k$, and $R_{g}$ is the radius of gyration of the

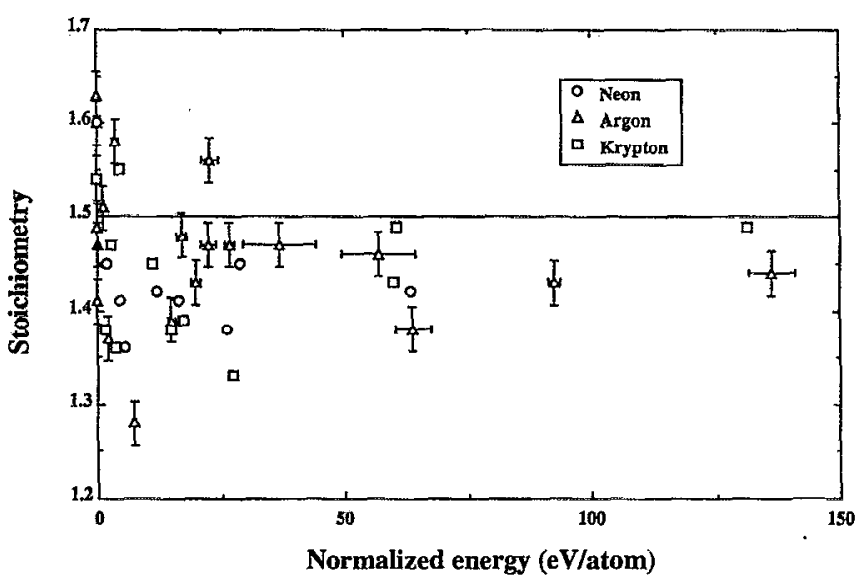

FIG. 1. Stoichiometry of alumina as a function of normalized energy and bombardment gas.

void, which is related to the void size ${ }^{24}$ by $R_{g}=R(3 / 5)^{1 / 2}$, $I(0)$ is the total intensity of $\mathrm{x}$ rays (at $k=0$ ) scattered from the primary beam $\left(I_{p}\right)$ by $\Delta n$ excess (deticit) electrons in the irradiated volume, $r_{e}$ is the classical electron radius (Thompson scattering factor), and $L$ is the sample-to-detector distance. By using the method of Kratky et $a .^{25}$ to determine the ratio of the scattered beam to the primary beam, $I(0) / Y_{p}$, the void density, $N$, can be determined from $\Delta n^{2}$ $=N \Delta \rho{ }^{2} V^{2} V_{s}$, where $\Delta \rho$ is the electron density difference between void and solid, $V$ is the void volume, and $V_{s}$ is the volume of the irradiated sample.

\section{RESULTS}

\section{A. Composition and microstructure}

Low energy ion bombardment with either $\mathrm{Ne}, \mathrm{Ar}$, or $\mathrm{Kr}$ has little effect on film stoichiometry as shown in Fig. 1. Throughout, error bars were included for only one data subset to avoid obscuring the data; errors for other subsets are comparable to those shown. The stoichiometry of the films varies between 1.3 and 1.6 with an average of 1.46 , and there is no correlation with normalized energy. Since depositions were conducted over a much narrower energy range than $R$ ratio range, the same figure indicates that there is no correlation with $R$ ratio. Attempts to correlate the stoichiometry with freshness of charge material were also unsuccessful. Nevertheless, the films are generally oxygen deficient, reflecting a loss of oxygen to the chamber during the deposition process. Ion bombardment also results in the incorporation of inert gas. Figure 2 shows that the inert gas concentration increases with increasing $E_{n}$ up to $\sim 20 \mathrm{eV} /$ atom, after which the argon and krypton concentrations saturate between 4 and 5 at.\%. Error bars on the vertical scale are controlled by the mass resolution of RBS, and etror bars on the horizontal scale are controlled by the variability in ion beam current and deposition rate during synthesis.

Although results are commonly reported as a function of normalized energy, $E_{n}$, the great majority of the variation is in the $R$ ratio which varies by a factor of 60 while the ion energy varies by only a factor of 2 . This point is emphasized 


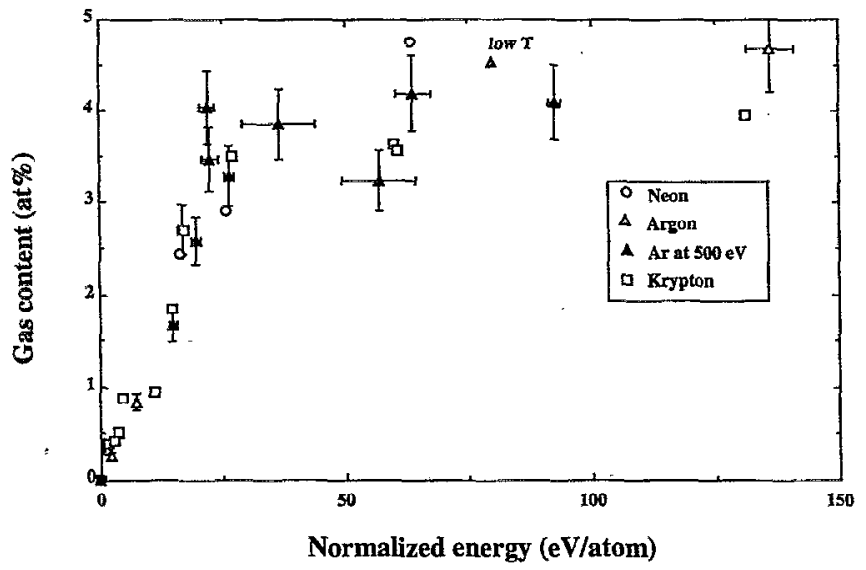

FIG. 2. Gas incorporation intö alumina films as a function of normalized energy and type of gas. Samples synthesized using $\mathrm{Ar}, E=500 \mathrm{eV}$, ion bombardment are shown as solid symbols.

by highlighting (using solid triangles), in the following graphs (as in Fig. 2), the results for Ar ion bombardment at a fixed energy $(500 \mathrm{eV})$ to show that the trend of this data subset is consistent with the overall data trend.

The film densities determined by RBS and thickness measurement (profilometry or ellipsometry) or by the index of refraction are shown in Fig. 3. The densities ranged from 2.6 to $3.1 \mathrm{~g} / \mathrm{cm}^{3}$ with an average of $2.84 \mathrm{~g} / \mathrm{cm}^{3}(72 \% \mathrm{TD})$ as determined from RBS and profilometry, $2.73 \mathrm{~g} / \mathrm{cm}^{3}(69 \%$ TD) from ellipsometry, and $3.24 \mathrm{~g} / \mathrm{cm}^{3}$ from the index of refraction. The large error bars and variability in results between techniques is due primarily to the imprecision of thickness measurements by profilometry. Presumably, the normalized energy range is below the critical energy threshold for densification of $\mathrm{Al}_{2} \mathrm{O}_{3}$, which for $\mathrm{ZrO}_{2}$ and $\mathrm{CeO}_{2}$ films was found to be $300-1000 \mathrm{eV} /$ atom. ${ }^{6,26}$

The microstructure of the oxide films synthesized by PVD is characterized by an amorphous structure with small

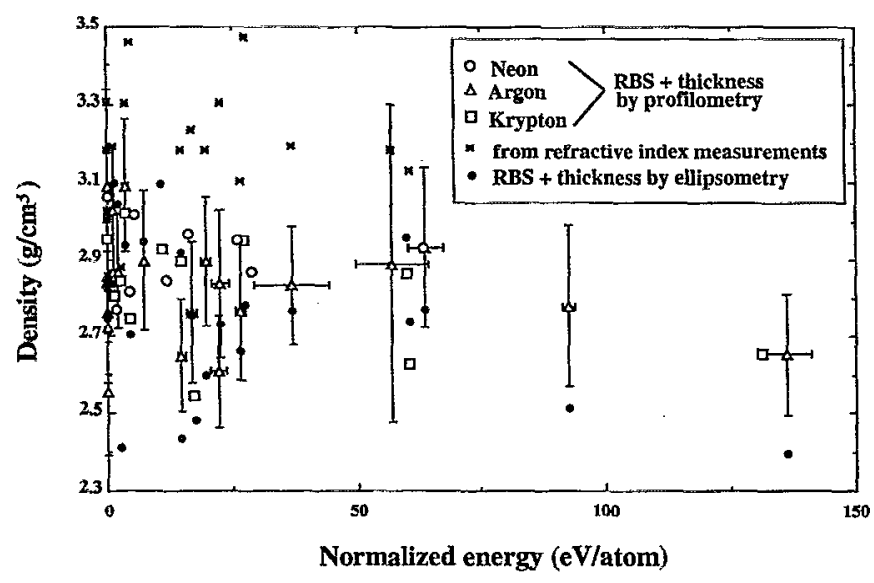

FIG. 3. Film density as a function of normalized energy and bombardment gas. Density is measured by either RBS and thickness as measured by profilometry (open symbols), RBS and thickness as measured by ellipsometry (solid symbol), or from refractive index measurements (asterisk).

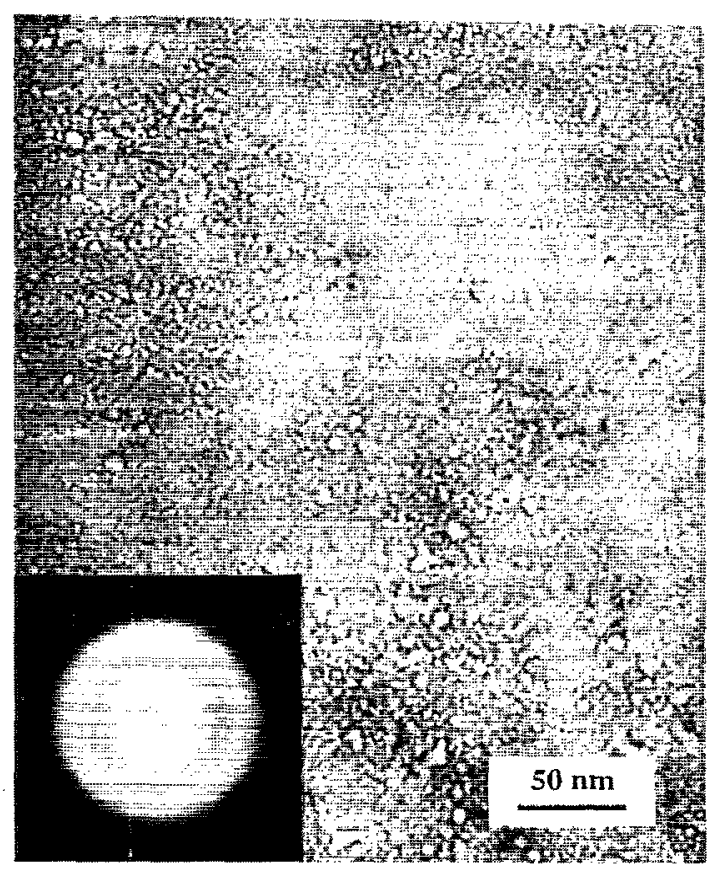

FIG. 4. Bright field electron micrograph and diffraction pattern showing amorphous structure of alumina deposited by IBAD using $\mathrm{Ar}, E=500 \mathrm{eV}$ and $R=0.18$.

amounts of crystalline phase, $4-5 \mathrm{~nm}$ in diameter, embedded in the amorphous matrix. Electron and x-ray diffraction of these films revealed interatomic distances consistent with $y$ $\mathrm{Al}_{2} \mathrm{O}_{3}$. Any amount of ion bombardment during deposition suppresses formation of the crystalline phase, resulting in purely amorphous oxide layers under all IBAD conditions. There is no evidence of a crystalline phase of $\mathrm{Kr}$ or Ar in any IBAD films.

TEM observations of a sample synthesized by IBAD (Ar) with $E_{n}=90 \mathrm{eV} /$ atom $(R=0.18)$ show a fairly uniform distribution of voids (Fig. 4), with an average radius of $3 \mathrm{~nm}$ and a number density of $3 \times 10^{18} \mathrm{~cm}^{-3}$, as determined by quantitative image analysis. Average void size and density were also determined by SAXS from plots of intensity vs $k^{2}$ for samples with $E_{n}$ values of 0,20 , and $50 \mathrm{eV} /$ atom (Ar) (Fig. 5). Direct comparison between TEM and SAXS results are made for the high $E_{n}$ sample. As shown in Table I, the void size as measured in SAXS is larger while the number density is smaller. Nevertheless, the resulting void volume is approximately the same and accounts for most of the $\sim 30 \%$ porosity reported earlier in the density measurements.

\section{B. Residual stress}

Residual stress as a function of normalized energy is plotted in Fig. 6 for all films. This data encompasses films formed by bombardment with $\mathrm{Ne}, \mathrm{Ar}$, and $\mathrm{Kr}$ and for film thicknesses ranging from 200 to $1200 \mathrm{~nm}$. As shown in Fig. 6, PVD samples (with no bombardment) exhibit a tensile stress of magnitude $0.4 \mathrm{GPa}$. Since the relative magnitudes of the coefficients of thermal expansion for $\mathrm{Al}_{2} \mathrm{O}_{3}$ and the 


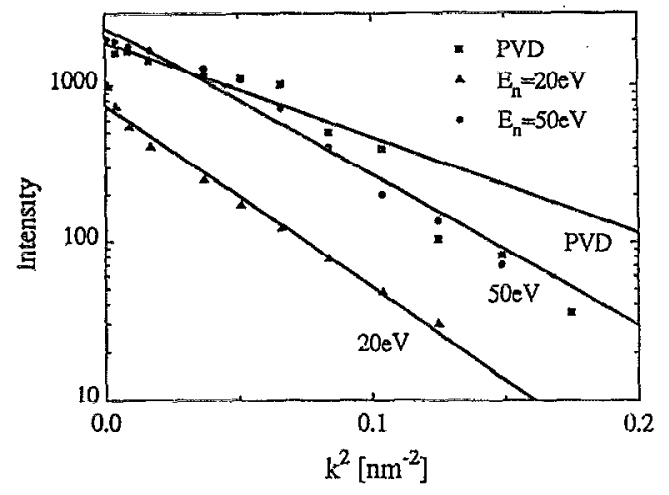

FIG. 5. Small angle $x$-ray scattering intensity as a function of the square of the diffraction vector, $k^{2}$ for three deposition conditions: PVD, IBAD $\left(E_{n}=20 \mathrm{eV} / \mathrm{atom}\right)$ and IBAD $\left(E_{n}=50 \mathrm{eV} /\right.$ atom $)$, both using $\mathrm{Ar}^{+}$.

cover slip dictate a small ( $<-0.07 \mathrm{GPa}$ ) but slightly compressive stress, the measured tensile stress must be primarily intrinsic in nature. Bombardment with increasing normalized energy leads to a decrease in the tensile stress and a crossover to a compressive stress state. The transition from tensile to compressive stress occurs at a very low normalized energy of between 1 and $3 \mathrm{eV} /$ atom. The maximum compressive stress, $-0.4 \mathrm{GPa}$, is attained at a normalized energy of about $20 \mathrm{eV} /$ atom, after which the stress changes only minimally. Over this thickness range, there is no dependence of residual stress on film thickness (Fig. 7).

\section{DISCUSSION}

The primary objective of this work is to gain an understanding of the residual stress behavior of the amorphous oxide films resulting from ion beam assisted deposition. Thus, we will focus on the departure of the stress state from the PVD reference point. This system is particularly interesting in that the direction of change is monotonic in the compressive direction. So, accepting a tensile stress state with no bombardment, the effect of ion bombardment is to drive the stress in the compressive direction. This behavior eliminates mechanisms which will only explain tensile stresses, such as grain growth and densification. They are also eliminated by default since the film is amorphous and no measurable densification occurs over the range of experimental parameters. Phase changes can also be eliminated since all ion bombarded films were observed to be amorphous. The exception is the PVD sample which contained a small volume fraction $(<10 \%)$ of crystalline material. However, any stress produced by a crystalline to amorphous transformation under

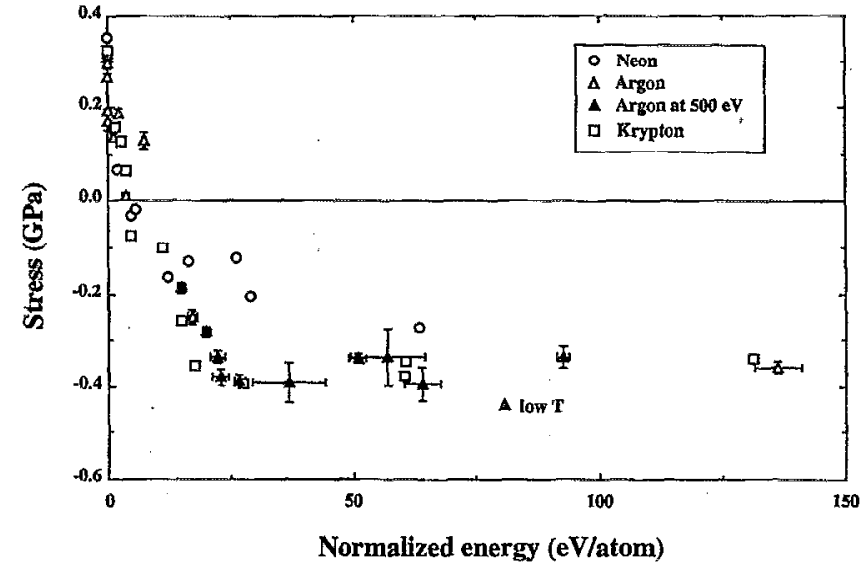

FIG. 6. Residual film stress as a function of normalized energy and bombardment gas. Samples synthesized using $500 \mathrm{eV}$ Ar ion bombardment are shown as solid symbols.

ion bombardment would be consistent with the observed compressive stress. Further, since all ion bombarded samples were amorphous, the effect of such a transformation is confined to very low values of $R$ or $E_{n}$.

This leaves mechanisms which depend on atomic rearrangements by ion bombardment and the effect of trapped gas. Both the thermal spike model and the recoil implantation model provide dependencies on the normalized energy. Equation (1) shows that for a given type of film, the recoil implantation model predicts that the stress should be proportional to the $R E^{1 / 2}$ and $\delta$, with all other quantities being constant. Since $\delta$ is a function of the $Z$ and $M$ of the film and the type of bombarding particle only, a change in the ion type should produce a different slope in a $\sigma$ vs $R E^{1 / 2}$ curve. This is shown in Fig. 8 for $\mathrm{Nc}$, Ar, and $\mathrm{Kr}$ ion bombardment. Equation (1) predicts that bombardment with $\mathrm{Kr}$ should result in a slope 0.83 times that of Ar, while bombardment with Ne should produce a slope 1.05 times that with Ar. A least squares fit to the experimental data over the range 0 to 1.2 $\mathrm{eV}^{1 / 2}$ shows that the data is indeed fit reasonably well with a linear dependence on $R E^{1 / 2}$. However, the slopes vary in the opposite direction with $\mathrm{Kr}$ having the greatest slope and $\mathrm{Ne}$ the smallest. Given the scatter in the data, and the relatively small $R^{2}$ values characterizing the fit, neither a verification nor a refutation of the model can be made on this point alone.

The thermal spike model, proposed as an explanation for the observed effect of ion bombardment on a transition in

TABLE I. Results of TEM and SAXS on $\mathrm{Al}_{2} \mathrm{O}_{3}$ films synthesized by IBAD.

\begin{tabular}{lccccc}
\hline \hline & & \multicolumn{3}{c}{ SAXS/TEM } \\
\cline { 4 - 6 } Deposition & $R$ ratio & $\begin{array}{c}\text { Normalized } \\
\text { energy, } E_{n} \\
(\mathrm{eV} / \text { atom })\end{array}$ & $\begin{array}{c}\text { Void size, } r \\
(\mathrm{~nm})\end{array}$ & $\begin{array}{c}\text { Void density, } N_{v} \\
\left(\mathrm{~cm}^{-3}\right)\end{array}$ & $\begin{array}{c}\text { Void fraction, } \\
\frac{\Delta V}{V}=\frac{4}{3} \pi r^{3} N_{v}\end{array}$ \\
\hline PVD & 0.0 & 0 & 4.1 & $1.1 \times 10^{18}$ & $0.32:$ \\
IBAD & 0.04 & 20 & 5.1 & $2.8 \times 10^{17}$ & 0.16 \\
IBAD & 0.1 & 50 & $4.7 / 3.0$ & $5.0 \times 10^{17} / 3 \times 10^{18}$ & $0.22 / 0.3$ \\
\hline \hline
\end{tabular}




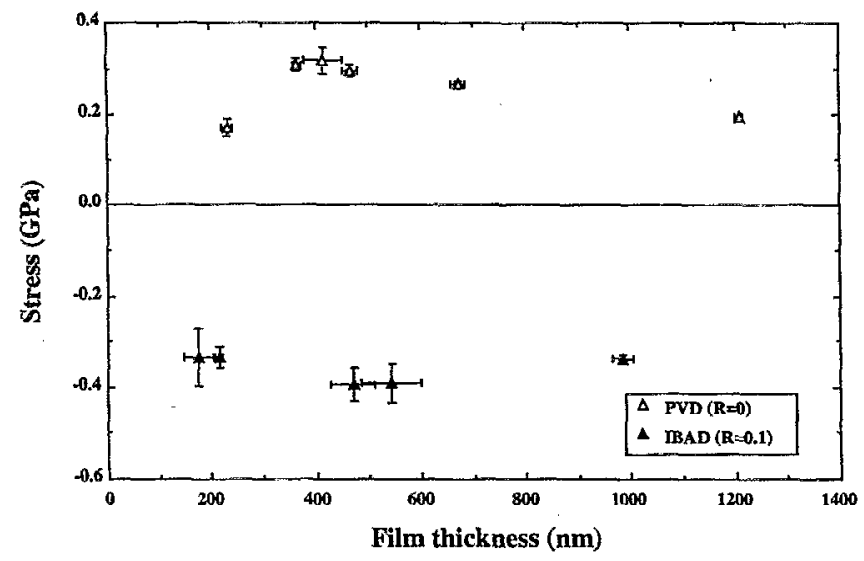

FIG. 7. Residual film stress as a function of film thickness as synthesized by PVD and $\operatorname{IBAD}(R=0.1)$.

adhesion by Hirsch and Varga, ${ }^{7}$ predicts that the critical current for transition should vary as $E^{-5 / 3}$, while their data follows an $E^{-3 / 2}$ behavior. Brighton and Hubler ${ }^{27}$ showed that the data are consistent with a binary collision cascade model in which the critical current varies with $E^{-1.59}$. Because of the narrow range over which energy is varied, our database is insufficient to verify this model. However, it is interesting to note that Hirsch and Varga also obtained a linear dependence of incorporated $\mathrm{Ar}$ on ion current density ( $R$ ratio), consistent with our results.

The chief problem in using either the thermal spike or atom peening models to explain our results lies in the saturation of the stress with increasing $E_{n}$, as shown in Fig. 6. This region was omitted in the plot of $\sigma$ vs $R E^{1 / 2}$ in Fig. 8 and is not predicted by either model. It is also coincident with an abrupt change in Ar gas incorporation at a normalized energy of $\sim 20$ eV/atom, as seen by comparing Figs. 2 and 6 . Below this transition, about $80 \%$ of incoming gas ions of any type are incorporated in the film (Fig. 9), after which the gas concentration saturates and the incorporation probability drops to zero. The variation of stress with incorporated gas is shown in Fig. 10. These data provide strong

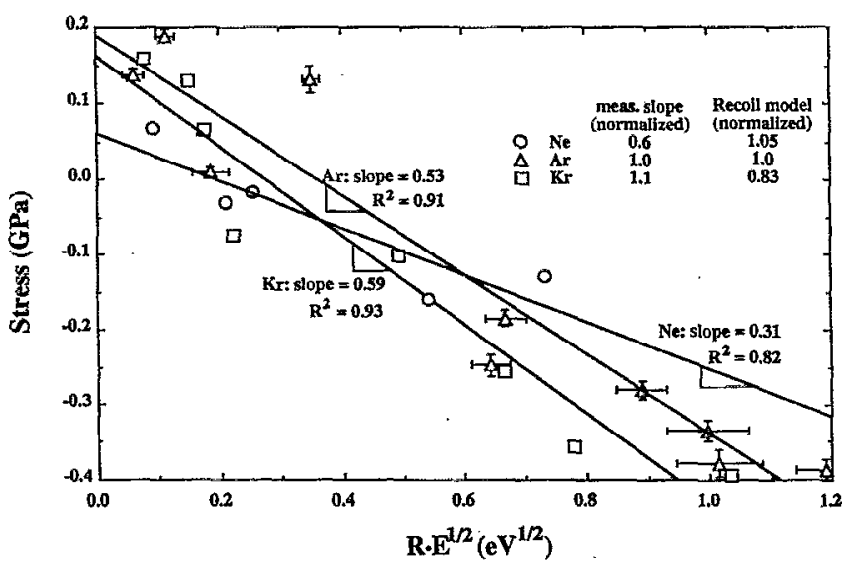

FIG. 8. Linear portion of the stress vs $R E^{1 / 2}$ plot for three different bombardment gasses.

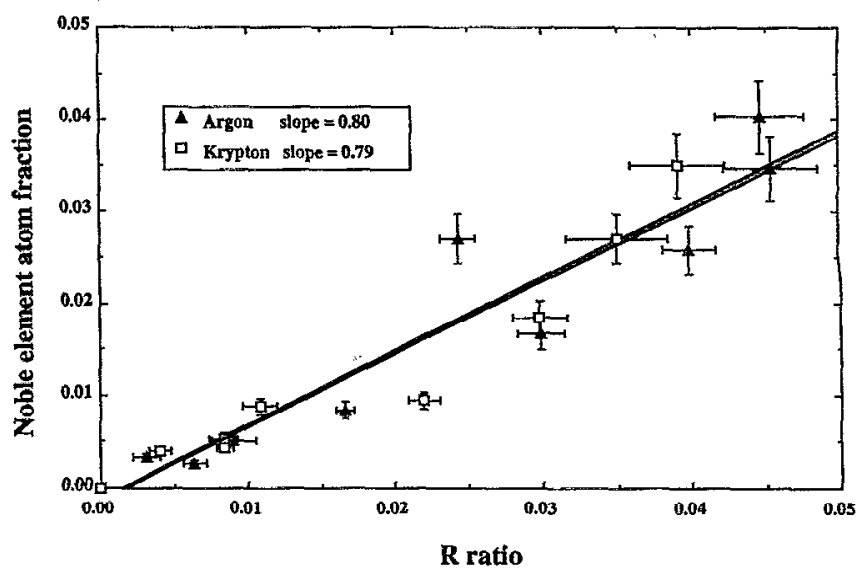

FIG. 9. Incorporated gas vs $R$ ratio for $\mathrm{Ar}$ and $\mathrm{Kr}$ ion bombardment up to $R=0.05$.

evidence that the incorporated gas plays a major role in the generation of the observed stresses, which is not considered in either the atom peening or thermal spike models. The mechanism may be due either to gas trapped in the matrix or gas agglomeration into voids observed to be present in these films.

The agglomeration of gas into voids has been shown to occur in the formation of $\mathrm{Al}^{13}$ and $\mathrm{TiN}^{28}$ films by IBAD. In our films, small angle $x$-ray scattering supports the existence of void-type defects of size $6-10 \mathrm{~nm}$ in diameter and in number densities $3-30 \times 10^{17} \mathrm{~cm}^{-3}$ and have been corroborated by measurements in transmission electron microscopy (Table I and Fig. 4). We assume that the void lattice is not appreciably changed between the PVD and IBAD samples and that the only difference is the incorporation of gas in the voids in the latter case. This assumption is supported by the consistency of the film density over all bombardment conditions, as well as the SAXS data. Although we have been able to verify the existence of $\mathrm{Kr}$ in the IBAD samples by energy

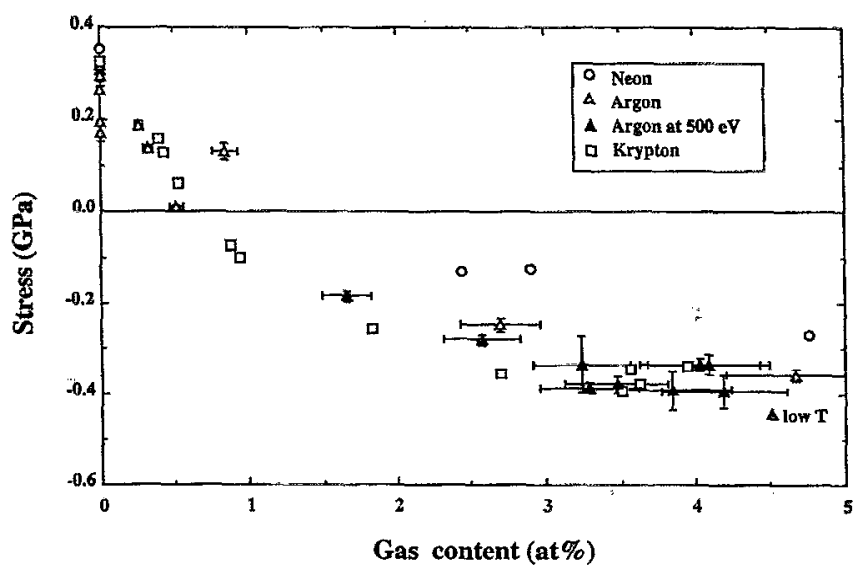

FIG. 10. Residual film stress as a function of gas content of the film and type of gas. Samples synthesized using $\mathrm{Ar}, E=500 \mathrm{eV}$, ion bombardment are shown as solid symbols. 
dispersive x-ray spectroscopy (EDS), the localization of $\mathrm{Kr}$ to the voids has not been established due to the inability to probe such a localized volume without interference from surrounding voids. It remains to be shown whether the gas in these voids can generate stresses of the magnitude measured.

This is done by modeling the film as an equivalent sphere in which the void and sphere radii are chosen so as to maintain the proper void volume fraction. The governing equation for the displacement, $u$, of the sphere boundary is

$$
\frac{d^{2} u}{d r^{2}}+\frac{2}{r} \cdot \frac{d u}{d r}-\frac{2 u}{r^{2}}=0 .
$$

The solution to the governing equation is

$$
u=A / r^{2}+B r
$$

with

$$
\begin{aligned}
& \epsilon_{r r}=\frac{\partial u}{\partial r}=-\frac{2 A}{r^{3}}+B, \\
& \epsilon_{\theta \theta}=\epsilon_{\phi \phi}=u / r=A / r^{3}+B,
\end{aligned}
$$

and

$$
\begin{aligned}
& \sigma_{r r}=2 \mu \epsilon_{r r}+\lambda\left(\epsilon_{r r}+2 \epsilon_{\theta \theta}\right), \\
& \sigma_{\phi \phi}=\sigma_{\theta \theta}=2 \mu \epsilon_{\theta \theta}+\lambda\left(\epsilon_{r r}+2 \epsilon_{\theta \theta}\right),
\end{aligned}
$$

where $\epsilon$ is strain, $\sigma$ is stress, $\mu$ is the shear modulus, $\lambda$ is the Lame coefficient, and $A$ and $B$ are constants. Applying boundary conditions of

(1) zero displacement of the surface of the equivalent sphere, $u=0$ at $r=r_{0}$, to account for the constraint of the film by the substrate which gives rise to a biaxial stress state, and

(2) equivalence of gas pressure and stress at the void surface, $\sigma_{r r}\left(r=r_{0}\right)=-p$, gives:

$$
\begin{aligned}
& B=-p /\left\{2 \mu\left[2(1 / f)^{3}+1\right]+3 \lambda\right\}, \\
& A=-B r_{0}^{3},
\end{aligned}
$$

and

$$
\sigma_{r r}=3 p(2 \mu+\lambda) /[4 \mu(1 / f)+(2 \mu+3 \lambda)],
$$

where $f=\left(r_{i} / r_{0}\right)^{3}$, is the volume fraction of void. Using a void volume fraction, $f=0.3, \nu=0.3$, and $E=150 \mathrm{MPa}^{24}$ gives $\mu=67 \mathrm{MPa}$ and $\lambda=101 \mathrm{MPa}$ for amorphous alumina ${ }^{29}$ and yields $\sigma_{r r} \approx-0.53 p$.

The number density of $\mathrm{Al}$ and $\mathrm{O}$ atoms in $\mathrm{Al}_{2} \mathrm{O}_{3}$ is calculated from the density of fully dense alumina, $3.97 \mathrm{~g} / \mathrm{cm}^{3}$. Using the atom fraction of $\mathrm{Ar}$ and $\mathrm{Kr}$ measured by RBS, and the measured void volume fraction $(\sim 0.3)$, the atom density of gas in the voids is determined assuming all gas is contained in voids. The results span the range from 20 to 200 $\mathrm{cm}^{3} / \mathrm{mol}$. The equations of state for compressible attracting spheres, compiled by Ronchi, ${ }^{30}$ are used to determine the gas pressure in the voids, which lies between 0.01 and $1.0 \mathrm{GPa}$. Substituting for $p$ into Eq. (13) and plotting the radial component of stress as a function of gas content yields the curves in Fig. 11 for $\mathrm{Ar}$ and $\mathrm{Kr}$. The radial component of stress represents a conservative estimate of the actual stress state in

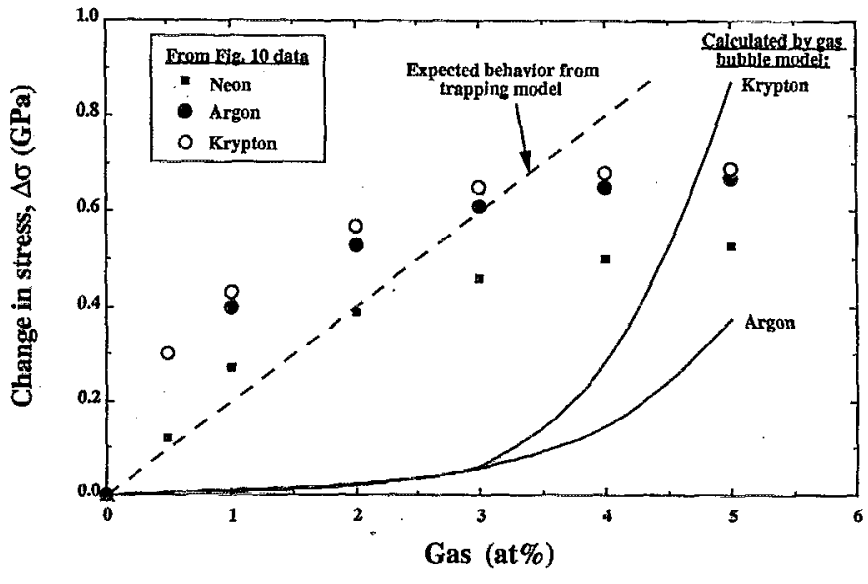

FIG. 11. Change in stress vs gas content. Symbols are measured values of stress and gas content. Lines represent calculations of the stress that would be generated if all of the gas were contained in the voids, trom Eq. (13).

the film. Note that the calculated stress versus gas curve has a positive second derivative due to the nonlinear behavior of compressibility with gas density. Note also that appreciable values of stress are not expected until the film contains $\geqslant 3$ at. $\%$ gas. The actual variation of stress with gas pressure is obtained from interpolation of Fig. 10. The data, however, follow a much different behavior with a large initial increase followed by saturation at $4-5$ at.\% gas. Because of the poor match between data and theory in both magnitude and curvature, it is unlikely that gas incorporation into voids alone can be responsible for the observed stresses. It is also noted that gas atoms are deposited approximately $1 \mathrm{~nm}$ below the surface during deposition. Since the spacing between voids is on average, $10 \mathrm{~nm}$, it is more likely that a gas atom will reach the surface than a void if it is not trapped in the matrix.

Molecular dynamics simulations of sputter deposited thin films have recently been shown to support a mechanism in which the tight packing of film atoms around injected gas atoms leads to a high compressive stress. ${ }^{14}$ The calculations were made on crystalline $\mathrm{Ni}$, and densification occurred along with an increase in the compressive stress, but.while density varied slowly with incident energy, stress changed rapidly. Incorporation of gas atoms into the matrix can result in the generation of a biaxial stress in the film proportional to the volume of the gas atom and its number density. While yielding in the film may explain the saturation in stress, it does not explain the coincident saturation in retained gas. Gas incorporation may be limited by release, either to the surface or to cracks. Observations of the surfaces indicate no evidence of cracking. The plateau in gas content with $R$ is most likely a result of trap saturation in the amorphous structure. At low $R$ ratios (Fig. 9), gas is accommodated at an $80 \%$ retention efficiency which drops to nearly zero at $R$ ratios above 0.05 . A possible explanation of this observation is that low concentrations of gas atoms are trapped at defects in the matrix until trap saturation occurs, after which the gas atoms are able to diffuse to the surface. This would give rise to a low apparent diffusivity at low $R$ (and low gas content) and a high apparent diffusivity at high $R$ (and high gas content), consistent with observation shown in Fig. 11. 
Trapping was investigated by performing a deposition on a copper specimen block cooled with liquid nitrogen, followed by annealing of the sample along with two others (one made with $\mathrm{Ar}$ and the other with $\mathrm{Kr}$ ) deposited at room temperature. Deposition at low temperature resulted in incorporation of 4.5 at. \% $\mathrm{Ar}$ and a residual stress of $-0.43 \mathrm{GPa}$, shown as the shaded triangle in Figs. 2, 6, and 10, both values only slightly higher than the respective depositions at room temperature (all were done at $500 \mathrm{eV}$ ). Annealing at $350{ }^{\circ} \mathrm{C}$ for $0.5 \mathrm{~h}$ and then again at $600{ }^{\circ} \mathrm{C}$ for $0.5 \mathrm{~h}$ resulted in no change in the amount or distribution of Ar or Kr, indicating that the gas is either strongly trapped in the lattice, or is released from traps to become immediately retrapped. Therefore, it is possible that the amount of gas incorporation is controlled by trapping rather than agglomeration into gas bubbles or limited diffusion kinetics.

\section{SUMMARY}

Bombardment of $\mathrm{Al}_{2} \mathrm{O}_{3}$ films, during deposition, using $\mathrm{Ne}, \mathrm{Ar}$, or $\mathrm{Kr}$ results in gas incorporation to $4-5$ at.\%. Gas is incorporated with an efficiency of $80 \%$ below $20 \mathrm{eV} /$ atom $(R \sim 0.05)$, above which gas incorporation ceases. Residual stress mirrors this behavior, rising from a high tensile stress into the compressive regime and saturating at $\sim 20 \mathrm{eV} /$ atom. There is no dependence of film stoichiometry on $R$ ratio or normalized energy and the stress is independent of film thickness between 200 and $1200 \mathrm{~nm}$. Microstructure consists of a void lattice where the voids are $3-5 \mathrm{~nm}$ in diameter and account for 15-30 vol \%. While the dependence of residual stress on normalized energy is consistent with the atom peening model, the saturation in stress and gas content and the relation between these variables cannot be explained by the model alone. Because the magnitude of the measured stress and its dependence on gas content are inconsistent with model predictions, it is unlikely that gas-filled bubbles are the primary source of stress. A probable source of stress is the gas residing in the matrix, the amount of which is controlled by trapping.

\section{ACKNOWLEDGMENTS}

The authors gratefully acknowledge the help of Ali Mashayekhi, Dr. Dave Hoffman, Dr. John Mansfield, Christine Kalnas, Hong Ji, and Professor Fred Terry in the preparation and analysis of samples. The authors also acknowledge the use of the facilities at the Scientific Research
Laboratory at the Ford Motor Company, and the Michigan Ion Beam Laboratory for Surface Modification and Analysis and the Electron Microbeam Analysis Laboratory of the University of Michigan.

${ }^{1}$ Surface Modification of Metals by Ion Beams, Proceedings of the Seventh International Conference on Surface Modification of Metals by Ion Beams, edited by F. A. Smidt, G. K. Hubler, and B. D. Sartwell (Elsevier Sequoia S. A., Lausanne, 1992).

${ }^{2}$ Surface Modification of Metals by Ion Beams, Proceedings of the Seventh International Conference on Surface Modification of Metals by Ion Beams, edited by M. Iwaki, B. D. Sartwell, and G. Dearnaley (Elsevier Sequoia S.A., Lausanne, in press).

${ }^{3}$ H. Windischmann, Crit. Rev. Solid State Mater. Sci. 17, 547 (1992).

${ }^{4}$ M. F. Doerner and W. D. Nix, Crit. Rev. Solid State Mater. Sci. 14, 225 (1988).

${ }^{5}$ E. Klokholm, J. Vac. Sci. Technol. 6, 138 (1969).

${ }^{6}$ F. M. D'Heurle and J. M. E. Harper, Thin Solid Films 171, 81 (1989).

${ }^{7}$ E. H. Hirsch and I. K. Varga, Thin Solid Films 69, 99 (1980).

${ }^{8}$ F. Seitz and J. S. Koehler, Solid State Phys. 69, 305 (1956).

${ }^{9}$ D. W. Hoffman and J. A. Thornton. Thin Solid Films 40, 355 (1977).

${ }^{10}$ H. Windischmann, J. Appl. Phys. 65, 1800 (1987).

${ }^{11}$ P. Sigmund, Topics in Applied Physics: Sputtering by Ion Bombardment, edited by R. Behrisch (Springer, Berlin, 1981), Vol. 47.

${ }^{12}$ J. A. Thornton, J. Tabock, and D. W. Hoffman, Thin Solid Films 64, 111 (1979).

${ }^{13}$ V. Dietz, P. Ehrhart, D. Guggi, H.-G. Haubold, W. Jäger, M. Prieler, and W. Schilling, Nucl. Instrum. Methods Phys. Res. B 59/60, 284 (1991).

${ }^{14}$ C. C. Fang, F. Jones, and V. Prasad, J. Appl. Phys. 74, 4472 (1993).

${ }^{15}$ D. W. Hoffman and M. R. Gaerttner, J. Vac. Sci. Technol. 17, 425 (1980).

${ }^{16}$ J. A. Thornton, J. Vac. Sci. Technol. 11, 666 (1974).

${ }^{17}$ L. R. Dolittle, Nucl. Instrum. Methods Phys. Res. B 9, 334 (1985).

${ }^{18}$ C. J. Brinker and S. P. Mukherji, Thin Solid Films 77, 141 (1981).

${ }^{19}$ J. A. Thornton, J. Tabock, and D. W. Hoffman, Thin Solid Films 64, 111 (1979).

${ }^{20}$ J. M. E. Harper, J. J. Cuomo, R. J. Gambino, and H. R. Kaufman, Nucl. Instrum. Methods Phys. Res. B 7/8, 886 (1985).

${ }^{21} \mathrm{~K}$. Kuwahara, T. Sumomogi, and M. Kondo, Thin Solid Films 78, 41 (1981).

22 J. A. Thornton and D. W. Hoffman, Thin Solid Films 171, 5 (1989).

${ }^{23}$ A. Guinier and G. Fournet, Small-Angle Scattering of $X$-Rays (Wiley, New York, 1956).

${ }^{24}$ G. Porod, "General Theory," in Simall-Angle X-ray Scattering, edited by O. Glatter and O. Kratky (Academic, London, 1982).

${ }^{25}$ O. Kratky, I. Pilz, and P. J. Schmitz, J. Colloid Interface Sci. 21, 24 (1966).

${ }^{26}$ D. S. Lee, J. Floro, D. J. Nikalsen, J. J. Cuomo, K. Y. Ahn, and D. A. Smith, J. Vac. Sci. Technol. A 3, 2121 (1985).

${ }^{27}$ D. R. Brighton and G. K. Hubler, Nucl. Instrum. Methods Phys. B 28, 527 (1987).

${ }^{28}$ P. R. Kazansky, L. Hultman, I. Ivanov, and J.-E. Sundgren, J. Vac. Sci. Technol. A 11, 1426 (1993).

${ }^{29}$ B. N. Lucas and W. C. Oliver, Material Research Society Symposium (Materials Research Society, Pittsburgh, PA, 1992), Vol. 239, pp. 337341.

${ }^{30}$ C. Ronchi, J. Nucl. Mater. 96, 314 (1981). 